\title{
Avaliação de cultivares de milho no agreste semi-árido de Pernambuco
}

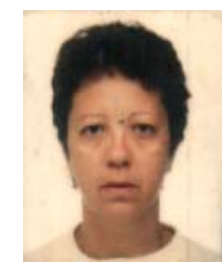

Marta M. A. do Nascimento1 ${ }^{1}$, José N. Tabosa² \& José J. Tavares Filho ${ }^{3}$

\author{
${ }^{1}$ IPA. Sitio Malhada de Pedra, CEP 55.000-000, Caruaru, PE. Telefax: 813711-2100. E-mail: martamancio@ig.com.br (Foto) \\ 2 IPA. Av. Gal. San Martin 1371, CEP 50761-000, Bonji, Recife, PE. Fone: (81) 445-2200, Ramal: 174, Fax: (81) 227-4017. \\ E-mail: tabosa@ipa.br \\ ${ }^{3}$ IPA. Email: jorge@ipa.br
}

Protocolo 70 - 10/5/2002 - Aprovado em 14/4/2003

\begin{abstract}
Resumo: Na localidade de São Bento do Una, Agreste Semi-árido de Pernambuco, foram avaliadas 12 cultivares de milho (híbridos e variedades) no triênio 1989/1991, com o objetivo de se identificar e avaliar o comportamento de cultivares de milho nessa região. 0 delineamento experimental foi de blocos ao acaso, com 12 tratamentos e três repetições. Avaliaram-se a produção de grãos, a altura da planta, o número de dias até a floração masculina, a altura de inserção da espiga e a população das plantas. Foi realizado um estudo de correlação entre os dados obtidos, cujos os resultados médios de produtividade de grãos variaram de 4.215 a $4.938 \mathrm{~kg} \mathrm{ha}^{-1}$, nos híbridos duplos XL-678 e BR-201, respectivamente. Quanto às variedades, a oscilação foi de 3.777 a $4.412 \mathrm{~kg} \mathrm{ha}^{-1}$, nos materiais BR-5028 e CMS-22, respectivamente. De maneira geral, houve supremacia de $13 \%$ em favor dos híbridos, com relação à produção de grãos, quando comparados com as variedades. O híbrido comercial (BR-201) apresentou-se $11 \%$ mais produtivo que a variedade mais produtiva (CMS-22) em relação à produtividade de grãos.
\end{abstract}

Palavras-chave: variedade, híbrido, correlação e produção de grãos

\section{Evalution of maize cultivars in the semi-arid agreste region of Pernambuco}

\begin{abstract}
At São Bento do Una locality of the Semi-arid "Agreste Region" of State of Pernambuco, Brazil, 12 maize cultivars were evaluated. The objetive of this study was to evaluate the performance of maize cultivars in this region. The experiment was carried out in randomized blocks with three replications. The evaluated parameters were: grain production, plant height, flowering, ear height and plant population. The grain production varied from 4,215 to $4,938 \mathrm{~kg} \mathrm{ha}^{-1}$ for Double hibrid 678, and BR-201 materials, respectively. In general, the hybrid materials produced $13 \%$ more. The varieties presented yield levels of $3,777 \mathrm{~kg} \mathrm{ha}^{-1}$ (BR-5028) to 4,412 $\mathrm{kg} \mathrm{ha}^{-1}$ (CMS-22). The BR-201 hybrid presented $11 \%$ increase compared to CMS-22 variety in terms of grain yield.
\end{abstract}

Key words: variety, hybrid, correlation, grain production

\section{INTRODUÇÃO}

O Nordeste brasileiro apresenta características bastante diferenciadas das demais regiões do país. O trópico semi-árido se ressente da contínua escassez de água em seu complexo planta-animal-homem. Tal fato resulta da distribuição espaçotemporal irregular das chuvas, da elevada evaporação e da baixa capacidade de retenção de umidade da grande maioria dos solos da região (Mafra, 1981).

O desenvolvimento de cultivares de milho, com altas produtividades de grãos, tem sido o objetivo principal dos melhoristas que trabalham com essa cultura. Segundo Vencovsky \& Torres (1988) as interações genótipo x ambiente influenciam as produções das cultivares de milho. A estabilidade da produção é o principal fator de recomendação de cultivares e híbridos (Johnson, 1978).

Segundo Johnson (1978) a produção agrícola na região semiárida do Nordeste brasileiro é prejudicada pelo fato dessa área ficar freqüentemente submetida aos rigores da seca. O milho é uma cultura tradicional na zona semi-árida, apesar de se tratar de um cereal sensível à seca (USAID, 1971). Não obstante, entre 1950 e 1975 ocorreu, no Nordeste, um aumento na área cultivada com milho em mais de duas vezes e meia (Johnson, 1978). Entre 1990 a 1998, a área média cultivada com milho em Pernambuco ficou em torno de 311 mil hectares (IBGE, 2002). A despeito disso, Maciel et al. (1986b) observaram, nos últimos anos, uma séria crise no abastecimento desse cereal no Estado de Pernambuco, em decorrência do crescimento da demanda 
de ração animal, principalmente pelo crescimento expressivo da avicultura. Estima-se que o Estado de Pernambuco importa cerca de $60 \%$ do milho consumido.

Os níveis médios de produtividade obtidos na região Nordeste se situam em torno de $500 \mathrm{~kg} \mathrm{ha}^{-1}$ de grãos (IBGE, 2002), valores considerados baixos, quando comparados aos de outras regiões do País. Pernambuco apresenta oito municípios com área colhida de 17 mil ha (Anuário Estatístico de Pernambuco, 1989).

A Empresa Pernambucana de Pesquisa Agropecuária (IPA), vem, com apoio da EMBRAPA, conduzindo o Ensaio Regional de Milho Nordeste com vistas a avaliar variedades de milho em seleção ou introduzidas, além de híbridos de outras regiões. Em fase de seleção e adaptação, na cultura do milho dificilmente a produção de grãos se mantém estável quando cultivada em ambientes diferentes. Isto significa que os efeitos genéticos e ambientais não são independentes, uma vez que as respostas fenotípicas dos genótipos podem diferir com as variações ambientais (Souza \& Vencovsky, 1989).

Desta forma, o objetivo deste trabalho foi identificar e avaliar o comportamento de cultivares de milho no Agreste Semi-árido de Pernambuco.

\section{MATERIAL E MÉTODOS}

O experimento foi conduzido na Estação Experimental da Empresa Pernambucana de Pesquisa Agropecuária, em São Bento do Una, Agreste Semi-Árido de Pernambuco, sob condições de sequeiro, no triênio 1989-1991. A caracterização edafoclimática e geográfica da localidade foi assim descrita por Encarnação (1980): latitude - 08³1'16"S; longitude - 36³6 $00^{\prime \prime}$ W; altitude - $650 \mathrm{~m}$; tipo climático - semi-árido megatérmico (D.d.A'a"), tipo de solo predominante, Regossolo. A precipitação ocorrida no ano de 1989, nos meses de abril, maio, junho e julho, foram de 127,5, 109,6, 49,0 e 105,9 mm, respectivamente. No ano de 1990, as precipitações ocorreram nos meses de abril $(68,1 \mathrm{~mm})$, maio $(71,2 \mathrm{~mm})$, junho $(67,0 \mathrm{~mm})$ e julho $(55,7$ $\mathrm{mm}$ ) e, no ano de 1991, nos meses de abril, maio, junho e julho, as precipitações foram, respectivamente, de 49,8, 58,2, 12,6 e $5,8 \mathrm{~mm}$. O delineamento experimental utilizado foi o de blocos ao acaso, com doze tratamentos e três repetições. Os tratamentos se constituíram de sete variedades de milho (BR-106, CMS35, CMS-33, BR-451, BR-5028, CMS-22 e BR-5037) e cinco híbridos duplos, também de milho (CONTI 133, AG-404, G- 500, BR-201 eXL-678).

As parcelas foram constituídas de quatro fileiras de $5 \mathrm{~m}$ de comprimento em que as duas fileiras centrais representaram a área útil, com espaçamento entre plantas de 1 x 0,5 m. Após o desbaste, foram deixadas duas plantas por cova e, em decorrência dos resultados das análises de solo (Tabela 1), usou-se a seguinte adubação: $50-60-30 \mathrm{~kg} \mathrm{ha}^{-1}$ de $\mathrm{N}$ (sulfato de amônio), $\mathrm{P}_{2} \mathrm{O}_{5}$ (super fosfato simples), $\mathrm{K}_{2} \mathrm{O}$ (cloreto de potássio), respectivamente, sendo $1 / 3$ do $\mathrm{N}$, o total de fósforo e o potássio aplicados, em fundação, e 2/3 do N em cobertura, 30 dias após o plantio. As variáveis avaliadas foram: produção de grãos, altura das plantas, altura de inserção da espiga, estande (população de plantas) e floração masculina. O plantio do experimento foi realizado no início da estação chuvosa de cada um dos três anos agrícolas.

Tabela 1. Resultados de análises químicas de solo da área experimental, em São Bento do Una, PE, no período estudado

\begin{tabular}{|c|c|c|c|c|c|}
\hline \multirow{2}{*}{ Ano } & $P$ & K & $\mathrm{Al}^{3+}$ & $\mathrm{Ca}^{2+}+\mathrm{Mg}^{2+}$ & \multirow{2}{*}{$\mathrm{pH}$} \\
\hline & \multicolumn{2}{|c|}{$\mathrm{mg} \mathrm{dm}{ }^{-3}$} & \multicolumn{2}{|c|}{$\mathrm{cmol}_{\mathrm{c}} \mathrm{dm}^{-3}$} & \\
\hline 1989 & 17,5 & 126 & 0,10 & 4,75 & 5,4 \\
\hline 1990 & $>80$ & 61 & 0,10 & 2,18 & 5,4 \\
\hline 1991 & $>80$ & 68 & 0,25 & 1,60 & 4,7 \\
\hline
\end{tabular}

Realizou-se a análise de variância de todas as variáveis, individualmente, para cada ano, e também a análise conjunta dos dados obtidos nos três anos estudados, além de se determinar a umidade de grãos, com vistas à correção do peso dos grãos para $15,5 \%$, para fins de análise de variância; por causa das variações existentes nos estandes de colheita, o peso de grãos foi corrigido com base no estande ideal, de acordo com a fórmula sugerida por Zuber (1942):

$$
\mathrm{PCC}=\mathrm{PC}[(\mathrm{H}-0,3 \mathrm{~F}) / \mathrm{H}-\mathrm{F}]
$$

em que:

$$
\begin{aligned}
& \text { PCC - peso de campo corrigido } \\
& \text { PC - peso de campo } \\
& \text { H - número total de plantas/parcela } \\
& \text { F - número de falhas }
\end{aligned}
$$

Calculou-se o coeficiente de correlação linear entre as variáveis avaliadas, conforme os procedimentos propostos por Gomes (1985).

\section{RESULTADOS E DISCUSSÃO}

Verifica-se, na Tabela 2, que a análise de variância dos dados de 1989 apresentou $\mathrm{F}$ altamente significativo em relação às variáveis: altura da planta, altura de inserção da espiga e floração e, também, quanto à população de plantas. Com relação à produção de grãos, não houve diferença significativa; contudo, destacaram-se os seguintes genótipos: BR 201, G- 500, BR 5037 e AG-404, que produziram 6.111, 5.804, 5.583, e $5.404 \mathrm{~kg} \mathrm{ha}^{-1} \mathrm{de}$ grãos, respectivamente. Embora os dados de floração tenham apresentado significação estatística, observou-se que o ciclo da cultura não influenciou a produção de grãos. Não se constatou significância da produção de grãos entre as cultivares estudadas, mas se verificou, no ano, uma distribuição normal de chuvas, aliada a um quantitativo substancial de água (392 mm) em comparação com o ano de 1991 (156,0 mm). De acordo com os dados obtidos em 1990, nas variáveis altura da planta e floração o $\mathrm{F}$ apresentou-se altamente significativo, mas tal fato não ocorreu em relação aos demais parâmetros. A cultivar mais produtiva foi a CONTI 133, com $5.733 \mathrm{~kg} \mathrm{ha}^{-1}$ de grãos. Vale frisar que todos os materiais apresentaram produtividade de grãos superiores a $4 \mathrm{t} \mathrm{ha}^{-1}$. Neste ano, apesar da precipitação pluvial apresentar-se baixa (262 $\mathrm{mm}$ ) a distribuição se comportou de forma normal favorecendo, assim, uma produtividade de grãos similar à obtida no ano de 1989. 
Tabela 2. Resultados médios obtidos, relativos às variáveis produção de grãos, altura de plantas, inserção de espiga, população de plantas e floração ${ }^{1}$

\begin{tabular}{|c|c|c|c|c|c|c|c|c|c|c|c|c|c|c|c|}
\hline \multirow[t]{2}{*}{ Cultivar } & \multicolumn{3}{|c|}{$\begin{array}{l}\text { Produtividade de } \\
\text { Grãos }\left(\mathrm{kg} \mathrm{ha}^{-1}\right)\end{array}$} & \multicolumn{3}{|c|}{$\begin{array}{l}\text { Altura Média da } \\
\text { Planta }(\mathrm{cm})\end{array}$} & \multicolumn{3}{|c|}{$\begin{array}{l}\text { Altura Média de } \\
\text { Inserção }(\mathrm{cm})\end{array}$} & \multicolumn{3}{|c|}{$\begin{array}{c}\text { População de } \\
\text { Planta x } 100 \\
\left(\mathrm{n}^{\mathrm{o}} \text { de planta } \mathrm{ha}^{-1}\right)\end{array}$} & \multicolumn{3}{|c|}{$\begin{array}{c}\text { Floração } \\
\left(\mathrm{n}^{\mathrm{o}} \text { de dias }\right)\end{array}$} \\
\hline & 1989 & 1990 & 1991 & 1989 & 1990 & 1991 & 1989 & 1990 & 1991 & 1989 & 1990 & 1991 & 1989 & 1990 & 1991 \\
\hline CMS-35 & 4113 & 5092 & $2223 c$ & 227ijk & 161cde & $138 b c$ & $88 \mathrm{cde}$ & 82 & $75 b$ & $37 \mathrm{ab}$ & 36 & $38 b$ & 54ghi & $57 \mathrm{ef}$ & 61de \\
\hline CONTI 133 & 5009 & 5733 & $2827 \mathrm{abc}$ & $243 \mathrm{ab}$ & $212 \mathrm{a}$ & $182 \mathrm{a}$ & $134 \mathrm{a}$ & 105 & $107 \mathrm{a}$ & $38 \mathrm{ab}$ & 39 & $40 \mathrm{a}$ & $66 a$ & $67^{\mathrm{a}}$ & $67 a$ \\
\hline CMS-33 & 4999 & 4219 & $2414 \mathrm{abc}$ & $174 \mathrm{jk}$ & $148 \mathrm{e}$ & $137 \mathrm{bc}$ & 86de & 80 & $73 b$ & $35 \mathrm{ab}$ & 35 & $39 a b$ & 53hi & $55 \mathrm{f}$ & $60 \mathrm{e}$ \\
\hline BR-201 & 6111 & 5462 & $3243 a$ & 204efghi & 170abcde & $144 b c$ & $98 \mathrm{cde}$ & 75 & $73 b$ & $38 \mathrm{ab}$ & 36 & $40 \mathrm{a}$ & 62abcde & 64abc & $66 a b c$ \\
\hline BR-451 & 4528 & 4933 & $2292 \mathrm{bcd}$ & 190hijk & 192abcd & $142 \mathrm{bc}$ & $91 \mathrm{cde}$ & 72 & $77 \mathrm{~b}$ & $38 \mathrm{ab}$ & 39 & $39 \mathrm{ab}$ & 58cdefgh & 59def & 61 bcde \\
\hline CMS-22 & 5104 & 4932 & $3201 \mathrm{ab}$ & 206defghi & 187abcde & $158 \mathrm{abc}$ & $99 \mathrm{cde}$ & 107 & $87 \mathrm{~b}$ & $39 a$ & 39 & $28 \mathrm{c}$ & 58 cdefgh & $60 \mathrm{cde}$ & $64 \mathrm{bcd}$ \\
\hline XL-678 & 5106 & 5054 & 2484abc & $235 \mathrm{abc}$ & 192abcd & $140 b c$ & $128 \mathrm{ab}$ & 102 & $78 b$ & $36 a$ & 39 & $40 \mathrm{a}$ & $65 \mathrm{ab}$ & $66 a b$ & $67 \mathrm{a}$ \\
\hline BR-5028 & 4231 & 4853 & $2249 c$ & $168 \mathrm{k}$ & $163 \mathrm{bcde}$ & $133 c$ & $68 c$ & 75 & $77 b$ & $27 \mathrm{c}$ & 34 & $38 b$ & $58 \mathrm{cdefgh}$ & $62 \mathrm{bcde}$ & $63 \mathrm{~cd}$ \\
\hline
\end{tabular}

* e $\mathrm{e}^{* *}$ Significativo aos níveis de 5 e $1 \%$, ns - não significativo

De acordo com os resultados obtidos em 1991, pelo teste $\mathrm{F}$ visualiza-se uma significação estatística em todas as variáveis em estudo, com destaque para as cultivares G-500, BR-201 e CMS-22, que apresentaram produtividades superiores a $3,0 \mathrm{t} \mathrm{ha}^{-1}$, com precipitação pluvial de apenas $156 \mathrm{~mm}$. Esta baixa precipitação pode ter influenciado o baixo nível de rendimento, quando comparado com os dados obtidos nos dois anos anteriores.

Com os dados obtidos nos três anos de experimentação, processou-se a análise conjunta com as médias de todas as variáveis estudadas (Tabela 3), já a análise de variância mostrou um $\mathrm{F}$ significativo em todas as variáveis, enquanto não foi detectada nenhuma significação estatística na interação cultivar $\mathrm{x}$ ano, considerando-se as variáveis avaliadas. Das 12 cultivares avaliadas, o teste de Duncan ao nível de 5\% de probabilidade indicou que os híbridos comerciais não diferiram quanto à

Tabela 3. Resultados médios ${ }^{1}$ relativos as variáveis produção de grãos (PG), altura da planta (AP), altura de inserção de espiga (AIE), produção de planta (PP) e floração (F)

\begin{tabular}{llllll}
\multicolumn{1}{c}{ Cultivar } & \multicolumn{1}{c}{$\begin{array}{c}\text { PG } \\
\mathrm{kg} \mathrm{ha}^{-1}\end{array}$} & $\begin{array}{c}\text { AP } \\
\mathrm{cm}\end{array}$ & $\begin{array}{c}\text { AIE } \\
\mathrm{cm}\end{array}$ & $\begin{array}{c}\mathrm{PP} \\
\times 1000 \mathrm{ha}^{-1}\end{array}$ & $\begin{array}{l}\mathrm{F} \\
\mathrm{n}^{\mathrm{o}} \text { dias }\end{array}$ \\
\hline BR-106 (V) & $4,292 \mathrm{abcd}$ & $187 \mathrm{bcd}$ & $94 \mathrm{bcd}$ & $39 \mathrm{ab}$ & $65 \mathrm{ab}$ \\
CMS-35 (V) & $3,809 \mathrm{bcd}$ & $154 \mathrm{ef}$ & $81 \mathrm{cde}$ & $37 \mathrm{ab}$ & $56 \mathrm{e}$ \\
CONTI-133 (HD) & $4,520 \mathrm{abcd}$ & $210 \mathrm{a}$ & $115 \mathrm{a}$ & $39 \mathrm{a}$ & $67 \mathrm{a}$ \\
CMS-33 (V) & $3,877 \mathrm{bc}$ & $158 \mathrm{f}$ & $80 \mathrm{de}$ & $36 \mathrm{abc}$ & $55 \mathrm{e}$ \\
AG-404 (HD) & $4,553 \mathrm{abc}$ & $198 \mathrm{bc}$ & $95 \mathrm{bc}$ & $38 \mathrm{ab}$ & $62 \mathrm{c}$ \\
G-500 (HD) & $4,579 \mathrm{ab}$ & $198 \mathrm{ab}$ & $102 \mathrm{~b}$ & $36 \mathrm{abc}$ & $66 \mathrm{ab}$ \\
BR-201 (HD) & $4,938 \mathrm{a}$ & $173 \mathrm{df}$ & $82 \mathrm{cde}$ & $38 \mathrm{ab}$ & $64 \mathrm{~b}$ \\
BR-451 (V) & $3,918 \mathrm{bcd}$ & $174 \mathrm{cdd}$ & $80 \mathrm{de}$ & $38 \mathrm{ab}$ & $58 \mathrm{~d}$ \\
CMS-22 (V) & $4,412 \mathrm{abcd}$ & $188 \mathrm{bcd}$ & $97 \mathrm{~b}$ & $35 \mathrm{bc}$ & $60 \mathrm{~cd}$ \\
XL-678 (HD) & $4,215 \mathrm{abc}$ & $189 \mathrm{bcd}$ & $102 \mathrm{~b}$ & $38 \mathrm{ab}$ & $66 \mathrm{ab}$ \\
BR-5028 (V) & $3,777 \mathrm{~d}$ & $154 \mathrm{ef}$ & $73 \mathrm{e}$ & $33 \mathrm{c}$ & $61 \mathrm{c}$ \\
BR-5037 (V) & $4,156 \mathrm{bcd}$ & $170 \mathrm{def}$ & $84 \mathrm{cde}$ & $37 \mathrm{ab}$ & $55 \mathrm{e}$ \\
\hline F & $*$ & $* *$ & $* *$ & $* *$ & $* *$ \\
\hline CV (\%) & 18,35 & 10,43 & 14,82 & 8,97 & 3,22 \\
\hline
\end{tabular}

${ }^{1}$ Média de 3 anos. As médias seguidas das mesmas letras não se diferem estatisticamente * e ** Significativo aos níveis 5 e $1 \%$ de probabilidade pelos teste de Duncan V - Variedade; HD - Híbrido duplo produtividade, com variações de $4.215 \mathrm{~kg} \mathrm{ha}^{-1}$ (XL-678) a 4.938 $\mathrm{kg} \mathrm{ha}^{-1}$ (BR-201). Fato análogo ocorreu com as variedades que produziram de $4.412 \mathrm{~kg} \mathrm{ha}^{-1}$ (CMS-22) a $3.777 \mathrm{~kg} \mathrm{ha}^{-1}$ (BR-5028). Estes resultados foram similares aos encontrados por Carvalho et al. (2000) em que a produtividade média para as cultivares foi de $4.301 \mathrm{~kg} \mathrm{ha}^{-1}$. No geral, os híbridos foram $13 \%$ mais produtivos que as cultivares, considerando-se a média de produção dos híbridos versus média de produção das variedades.

Conquanto seja um dado experimental, a produtividade de $4.938 \mathrm{~kg} \mathrm{ha}^{-1}$ (BR-201) corresponde a cerca de sete vezes a produtividade média desta região, que é de $700 \mathrm{~kg} \mathrm{ha}^{-1}$. Segundo relato de Paterniani (1988) o milho, dependendo do genótipo, responde com maior ou menor intensidade aos diferentes ambientes; este valor, contudo, não representa uma vantagem nítida em produção de grãos, em favor dos híbridos.

O estudo da correlação entre as variáveis revelou efeito significativamente positivo entre a floração e a produção, constatado na média dos três anos considerados no estudo (Tabela 4). As precipitações pluviais de 1989, 1990 e 1991, foram de 392, 262 e $156 \mathrm{~mm}$, respectivamente. Os diferentes comportamentos, aptidões e prováveis adaptações existentes nos

Tabela 4. Coeficientes de correlação linear obtidos entre as variáveis (média de 3 anos) estudadas, em São Bento do Una

\begin{tabular}{|c|c|c|c|c|}
\hline Parâmetro & $\begin{array}{c}\text { Altura } \\
\text { da Planta } \\
\text { cm }\end{array}$ & $\begin{array}{c}\text { Altura } \\
\text { de Inserção } \\
\text { cm }\end{array}$ & $\begin{array}{c}\text { População } \\
\text { x } 1000 \\
\text { plantas ha-1 }\end{array}$ & $\begin{array}{l}\text { Floração } \\
\mathrm{n}^{0} \text { de dias }\end{array}$ \\
\hline $\begin{array}{l}\text { Produtividade } \\
\mathrm{kg} \mathrm{ha}^{-1}\end{array}$ & $0,7551 * *$ & $0,6435^{* *}$ & $0,5836^{*}$ & $0,7310 * *$ \\
\hline $\begin{array}{l}\text { Altura da } \\
\text { planta - cm }\end{array}$ & & $0,9236^{* *}$ & $0,5953^{\mathrm{NS}}$ & $0,7662 * *$ \\
\hline $\begin{array}{l}\text { Altura de } \\
\text { inserção } \\
\text { de espiga }-\mathrm{cm}\end{array}$ & & & $0,4800^{\mathrm{NS}}$ & $0,7026^{* *}$ \\
\hline $\begin{array}{l}\text { População } \\
\text { x } 1000 \\
\text { plantas ha- }\end{array}$ & & & & $0,3477^{\mathrm{NS}}$ \\
\hline
\end{tabular}


materiais avaliados, são devidos, certamente, ao fato dos híbridos e as variedades apresentarem diferenças no potencial para expressar o caráter produção de grãos. Paterniani (1988) afirma que uma das características de enorme importância é a variação climática, notadamente da pluviosidade, variação que ocorre de ano para ano e no mesmo ano, de local para local. Portanto, deve ser considerada, pelo melhoramento genético, a imprevisibilidade da variação climática na agricultura tropical, sobretudo em condições de sequeiro.

A variável produção de grãos apresenta correlações positivas e significativas, quando relacionada com as demais variáveis em estudo.

Estudando estabilidade de cultivares de milho no Nordeste brasileiro, Carvalho et al. (2000) observou que a variedade BR 106 mostrou estabilidade neste ambiente. Os sete materiais mais produtivos (BR-201, G-500, AG-404, CONTI 133, BR-106, XL-678 e CMS-22) todos exibindo produtividade acima de $4 \mathrm{t}$ $\mathrm{ha}^{-1}$ de grãos, demoraram para atingir o florescimento, quando comparados com o demais (Tabela 3). Resultados similares foram encontrados por Maciel et al. (1986a) em ambientes em que a população de plantas foi reduzida. Portanto, houve interferência na produção das variáveis relacionadas à floração e população de plantas. Esta condição se relaciona, provavelmente, à natureza de cada material avaliado.

\section{CONCLUSÕES}

1.Os híbridos comerciais BR-201, G500, AG-404, CONTI 133, X- 678 e as variedades CMS-22 e BR-106 apresentaram maior produtividade de grãos em relação aos demais híbridos e variedades estudadas, sob condições de sequeiro, no Agreste Semi-árido de Pernambuco.

2.Os híbridos comerciais apresentaram média de produtividade $13 \%$ a mais em relação às variedades estudadas.

\section{LITERATURA CITADA}

Anuário Estatístico de Pernambuco, Recife: CONDEPE, v.35, 1989.

Carvalho, H.W.L. de; Cardoso, M.J.; Oliveira, A.C.; Santos, M.X. dos; Santos, D.M. dos; Tabosa, J.N.; Lira, M.A.; Carvalho, B.C.L. de; Sampaio, G.V.; Dourado, V.V.; Oliveira, J.S. de; Brito, A.R. de M.B.; Tavares, J.A.; Nascimento, M.M.A. do; Tavares Filho, J.J.; Souza, E.M. de. Recomendações de culvitares de milho no Nordeste brasileiro: ensaios realizados no ano agrícola de 2000/2001. Aracaju: EMBRAPA, 2002, 8p. Comunicado Técnico, 1
Carvalho, H.W.L. de; Leal, M. de L. da S.; Santos, M.X. dos; Cardoso, M..J.; Monteiro, A.A.T.; Carvalho, B.C.L de. Estabilidade de cultivares de milho no nordeste brasileira. Pesquisa Agropecuária Brasileira, Brasília, v.35, n.9, p.17731781,2000a.

Carvalho, H.W.L. de; Leal, M. de L. da S.; Santos, M.X. dos; Cardoso, M..J.; Monteiro, A.A.T.; Tabosa, J.N. Adaptabilidade e estabilidade de cultivares de milho no nordeste brasileira. Pesquisa Agropecuária Brasileira, Brasília, v.35, n.6, p.1115-1123, 2000b.

Encarnação, C.R.F. da. Observações meteorológicas e tipos climáticos das Unidades e Campos Experimentais da Empresa IPA. Recife: IPA, 1980. 110p.

Gomes, F.P. Curso de estatística experimental. 10.ed. Piracicaba: ESALQ, 1985.466p.

IBGE - Instituto Brasileiro de Geografia e Estatística. Estatística da agropecuária de Pernambuco. Recife, 1986. 130p.

Johnson, D. Sorgo granífero no Nordeste do Brasil. Revista Econômica do Nordeste, Fortaleza, v.9, n.4, p.403 -500, 1978.

Maciel, G.A.; Melo, J.N.; Lira, M. de A.; Tavares Filho, J.J.; Tabosa, J.N. Estudo do comportamento de cultivares de milho nas condições do submédio São Francisco. Pesquisa Agropecuária Pernambucana, Recife, v.6, n. especial, p. 6977, 1986.

Maciel, G.A.; Tabosa, J.N.; Santos, J.P.O.; Lira, M. de A. Identificação de cultivares de milho para o Semi-Árido de Pernambuco. In: Congresso Nacional de Milho e Sorgo, 15. 1984, Maceió. Anais... Brasília: EMBRAPA-DDT, 1986. p.207216. EMBRAPA - CNPMS. Documentos, 5

Mafra, R.C. Agricultura de sequeiro no trópico semi-árido: um delineamento de compromisso para a pesquisa. Recife: Secretaria de Agricultura de Pernambuco/ IPA, 1981.59p.

Paterniani, E. Interação genótipo $\mathrm{x}$ ambiente em climas tropicais e subtropicais. In: Congresso Nacional de Milho e Sorgo, 16, 1986, Belo Horizonte. Anais... Sete Lagoas: EMBRAPA, 1988. p.378-382.

Souza J.R.C.L.; Vencovsky, R. Covariância entre parentes na presença da interação genótipo $\mathrm{x}$ ambiente. In: Simpósio de Estatística Aplicada à Experimentação Agronômica, 3, 1989, Lavras. Resumos... Lavras: ESAL, 1989. p.50-51.

USAID. Improving farm production in tropical and subtropical regions of limited rainfall. Washington: Agriculture Tecnology for Developing Countries, 1971.25p. USAID. Technical Bulletin, 4

Vencovsky, R.; Torres, R.A.A. Estabilidade geográfica e temporal de algumas cultivares de milho. In: Congresso Nacional de Milho e Sorgo, 16, 1986, Belo Horizonte. Anais... Sete Lagoas: EMBRAPA-CNPMS, 1988. p.294-299.

Zuber, M.S. Relative efficiency of incomplete block designs using corn uniformity trial data. Journal of the American Society of Agronomy, Madison, v.34, p.30-47, 1942. 